\title{
Geometric Hyperplanes of the Dual Half-Spin Geometry
}

\author{
Abdelsalam Abou Zayda \\ Mathematics Department, Alaqsa University \\ Gaza, Palestine \\ E-mail: zayda8@hotmail.com
}

\begin{abstract}
We will present two types of geometric hyperplanes of the dual half-spin geometry $D_{5,3}$, the class of subspaces of kind $p^{\perp}$ ( $p$ is a point in $D_{5,3}$ ) and substructures called Shult sets are determined to be hyperplanes of such geometry. Moreover we construct a binary constant weight code using the hyperplanes of the geometry.
\end{abstract}

Keywords: Hyperplanes, Shult set, Strong parapolar, Constant-weight code

\section{Introduction}

In a certain class of point-line geometries the geometric hyperplanes were classified. In [Bruyn, B. D. \& Pralle, H., 2007] the hyperplanes of DH(5,4) were characterized. In [Bruyn, B. D., 2007, p307] and [Bruyn, B. D., 2007, p309] All hyperplanes of DW(2n-1,q), $q \neq 2$ and DW $(5,2 \mathrm{~h})$ are determined. In this paper we presented two types of hyperplanes of the dual half-spin geometry $D_{5,3}(\mathrm{q})$ and we constructed a new family of binary constant-weight codes using such hyperplanes. First we present some definition of terminology's that will be used. For most of the following definitions see [Buekenhout, 1974]. Given a set $I$, a geometry $\Gamma$ over $I$ is an ordered triple $\Gamma=(X, *, D)$, where $X$ is a set, $D$ is a partition $X_{i}$ of $X$ indexed by $I, X_{i}$ are called components, and $*$ is a symmetric and reflexive relation on $X$ called incidence relation such that: $\mathrm{x} * \mathrm{y}$ implies that either $\mathrm{x}$ and $\mathrm{y}$ belong to distinct components of the partition of $\mathrm{X}$ or $\mathrm{x}=\mathrm{y}$. Elements of $\mathrm{X}$ are called objects of the geometry, and the objects within one component $X_{i}$ of the partition are called the objects of type $i$. The subscripts that index the components are called types. The obvious mapping $\tau: X \longrightarrow I$, which takes each object to the index of the component of the partition containing it is called the type map $\tau$. A point-line geometry $(P, L)$ is simply a geometry for which $|I|=2$, one of the two types is called points; in this notation the points are the members of $P$, and the other type is called lines. Lines are the members of $L$. If $p \in P$ and $\ell \in L$, then $p * \ell$ if and only if $p \in \ell$. In point-line geometry $(P, L)$, we say that two points of $P$ are collinear if and only if they are incident with a common line. (We use the symbol $\sim$ for collinear). The singular rank of a space $\Gamma$ is the maximal number $n$ (possibly $\infty$ ) for which there exist a chain of distinct subspaces $\emptyset=X_{0} \subset X_{1} \subset \cdots \subset X_{n}$ such that $X_{i}$ is singular for each $i, X_{i} \neq X_{j}, i \neq j$. For example rank $(\emptyset)=-1, \operatorname{rank}(p)=0$ where $p$ is a point and $\operatorname{rank}(\ell)=1$ where $\ell$ a line.

$x^{\perp}$ means the set of all points in $P$ collinear with $x$, including $x$ itself. A subspace of a point-line geometry $\Gamma=(P, L)$ is a subset $X \subseteq P$ such that any line which has at least two of its incident points in $X$ has all of its incident points in $X$. $<X>$ means the intersection over all subspaces containing $X$, where $X \subseteq P$. Lines incident with more than two points are called thick lines, those incident with exactly two points are called thin lines. In a point-line geometry $\Gamma=(P, L)$, a path of length $n$ is a sequence of $n+1\left(x_{0}, x_{1}, \cdots, x_{n}\right)$ where, $\left(x_{i}, x_{i}+1\right)$ are collinear, $x_{0}$ is called the initial point and $x_{n}$ is called the end point. A geodesic from a point $x$ to a point $y$ is a path of minimal possible length with initial point $x$ and end point $y$. We denote this length by $\mathrm{d}(x, y)$, the length of the geodesic from $x$ to $y$ is called the distance between $x$ and $y$. The diameter of the geometry is the maximal distance of points. A geometry is called connected if and only if for any two of its points there is a path connecting them. A subset $X$ of $P$ is said to be convex if $X$ contains all points of all geodesics connecting two points of $X$. A polar space is a point-line geometry $\Gamma=(P, L)$ satisfying the Buekenhout-Shult axiom: For each point-line pair $(p, l)$ with $p$ not incident with $\ell ; p$ is collinear with one or all points of $l$, that is $\left|p^{\perp} \cap \ell\right|=1$ or else $p^{\perp} \supset \ell$. Clearly this axiom is equivalent to saying that $p^{\perp}$ is a geometric hyperplane of for every point $p \in P$. A point-line geometry $\Gamma=(P, L)$ is called a projective plane if and only if it satisfies the following conditions [Cooperstein, 1977]:

i- $\Gamma$ is a linear space; every two distinct points $x, y$ in $P$ lie exactly on one line,

ii- every two lines intersect in one point,

iii- there are four points no three of them are on a line.

A point-line geometry $\Gamma=(P, L)$ is called a projective space if the following conditions are satisfied:

i- every two points lie exactly on one line (linear),

ii- if $\ell_{1}, \ell_{2}$ are two lines with $\ell_{1} \cap \ell_{2} \neq \phi$, then $\left\langle\ell_{1}, \ell_{2}\right\rangle$ is a projective plane. $\left(\left\langle\ell_{1}, \ell_{2}\right\rangle\right.$ means the smallest subspace of $\Gamma$ containing $\ell_{1}$ and $\ell_{2}$. 
A point-line geometry $\Gamma=(P, L)$ is called a parapolar space if and only if it satisfies the following properties:

i- $\Gamma$ is connected gamma space,

ii- for every line $\ell ; \ell^{\perp}$ is not a singular subspace,

iii- for every pair of non-collinear points $x, y ; x^{\perp} \cap y^{\perp}$ is either empty, a single point, or a non-degenerate polar space of rank at least 2 .

If $x, y$ are distinct points in $P$, and if $\left|x^{\perp} \cap y^{\perp}\right|=1$, then $(x, y)$ is called a special pair, and if $x^{\perp} \cap y^{\perp}$ is a polar space, then $(x, y)$ is called a polar pair or ( a symplectic pair). A parapolar space is called a strong parapolar space if it has no special pairs.

A subspace of a point line geometry $\Gamma=(P, L)$ is called a geometric hyperplane if it is a proper subspace containing at least one point of every line. Shult set. A subset $S$ of a geometry of diameter $n$ is called a partial Shult set if the distance between any pair of points of $S$ is $n$. If the maximum is achieved then $S$ is called a Shult set.

\section{Definition of Dual half-spin geometry $D_{5,3}$}

(For the graph of $D_{5,3}$ see figure 1 at the end of the paper).

Consider the classical polar space $\Delta=\Omega^{+}(10, F)$ that comes from a vector space of dimension 10 over a finite field $F=G F(k)$ with a symmetric hyperbolic bilinear form. The two classes $M_{1}, M_{2}$ consist of maximal totally isotropic 5dimensional subspaces. Two 5-subspaces fall in the same class if their intersection is of odd dimension. The geometry of type $D_{5,3}(F)$ is the point-line geometry $(P, L)$, whose set of points $P$ is corresponding to the class $S_{3}$ that is: the collection of all totally isotropic 3-dimensional subspaces of the vector space $V$, and whose lines are corresponding to the collection of all 5-dimensional subspaces of the vector space $V$ that are fall in the class $M_{1}$. A point $C$ is incident with a line $B$ if and only if $C \subset B$ as a subspaces of $V$. To define the collinearity, let $C_{1}$ and $C_{2}$ be two point (the points are the T.I 3-spaces), then $C_{1}$ is collinear to $C_{2}$ if and only if the intersection of $C_{1}$ and $C_{2}$ is a T.I 1-dimensional space. This intersection in addition to the complement of $C_{1}$ and $C_{2}$ must form a T.I 5-dimensional space. The elements of the class $M_{2}$ are geometries of type $A_{4,3}(F)$.

The symplecta of $D_{5,3}(F)$ are the Grassmannians of type $A_{3,2}(F)$ that are corresponding to the collection of TI 2 dimensional spaces.

Notation. Let the map $\Psi: P \rightarrow V$ defined above, i.e., $\Psi(p)$ is the T.I. 3-dimensional subspace corresponding to the point $p$. We will use $\Psi$ for the rest of the geometry; for example $\Psi\left(D_{4,2}\right)$ is the T.I. 1-dimensional subspace corresponding to a geometry of type $D_{4,2}$. The inverse map $\Psi^{-1}$ will be used for the inverse; for example $\Psi^{-1}(C)$ is the point corresponding to the T.I. 3-dimensional subspace $C$. The following two theorems was presented and proved in [Abdelsalam, 2007].

Theorem 2.1 [Abdelsalam, 2007]. Let $\Gamma=(P, L)$ be a point-line geometry of type $D_{5,3}$, then the following are satisfied:

P1 $\Gamma$ is a strong parapolar space of diameter 4.

P2 The symlecta of the geometry are of type $A_{3,2}$.

P3 If $(p, S)$ is a pair of non-incident point-symplecton, then $\operatorname{rank}\left(p^{\perp} \cap S\right)=-1,0,2$.

P4 If $S_{1}$ and $S_{2}$ are two different symplecta of $D_{5,3}$, then $\operatorname{rank}\left(S_{1} \cap S_{2}\right)=-1,0$.

Theorem 2.2 [Abdelsalam, 2007]. Let $\Gamma=(P, L)$ be a point-line geometry of type $D_{5,3}$, then the following are satisfied:

i. Let $(p, S)$ be a non-incidence pair of a point $\mathrm{p}$ and a symplecton $S$. Then $\operatorname{rank}\left(p^{\perp} \cap S\right)=-1,0$ or 2 .

ii. Let $S_{1}$ and $S_{2}$ be two symplecta in $D_{5,3}(F)$. Then $\operatorname{rank}\left(S_{1} \cap S_{2}\right)=-1$ or 0 .

\section{The main result}

We will determine two types of hyperplanes for the dual half-spin geometry $D_{5,3}$, theorem 3.1 is to prove that the first kind of hyperplanes is of the form $p^{\perp}$ for any point $p$. In Theorem 3.2 we show that the second type of hyperplanes is Shult sets.

Theorem 3.1. Let $p$ be a point in the geometry $D_{5,3}$, then $p^{\perp}$ is a geometric hyperplane of $D_{5,3}$.

proof. Let $(p, \ell)$ be a non incident pair of a point and a line. We prove that $\ell$ intersects $p^{\perp}$ nontrivially, so there are three cases for the intersection of $\Psi(p)=<x_{1}, x_{2}, x_{3}>$ and $\Psi(\ell)=<y_{1}, y_{2}, y_{3}, y_{4}, y_{5}>$ : 
1- $\Psi(p) \cap \Psi(l)=2$-space, say $<x, y>$, where $x=x_{1}=y_{1}, y=x_{2}=y_{2}$ and $x_{3}^{\perp} \cap<x, y, y_{3}, y_{4}, y_{5}>=<x, y, y_{3}, y_{4}>$. Now we take a point $r$ such that $\Psi(r)=<x, y_{3}, y_{4}>$. Since $\Psi(p) \cap \Psi(r)=1$-space and $<x, y, x_{3}, y_{3}, y_{4}>$ form a totally isotropic 5-space. Then $r$ is a point in $\ell$ and collinear to $p$, so $r \in p^{\perp}$ and $\ell \cap p^{\perp}=r$.

2- $\Psi(p) \cap \Psi(\ell)=1$-space, say $<x>$, where $x=x_{1}=y_{1}$ and $x_{2}^{\perp} \cap<x, y, y_{3}, y_{4}, y_{5}>=<x, y_{2}, y_{3}, y_{4}>, x_{3}^{\perp} \cap<$ $x, y, y_{3}, y_{4}, y_{5}>=<x, y, y_{2}, y_{3}, y_{5}>$. Let $s$ be a point in $\ell$ such that $\Psi(s)=<x, y_{2}, y_{3}>$, then $\Psi(p) \cap \Psi(s)=1$-space and $<x, x_{2}, x_{3}, y_{2}, y_{3}>$ form a totally isotropic 5-space. Then $p$ is collinear to $s$ and $\ell \cap p^{\perp}=s$.

3- $\Psi(p) \cap \Psi(\ell)=0$-space, then $\Psi(p)$ is contained in a maximal totally isotropic 5 -space $D=<x_{1}, x_{2}, x_{3}, u, v>$. Since the two maximal totally isotropic 5-spaces $\Psi(\ell)$ and $D$ fall in the same class of the classical finite polar space $\Omega^{+}(10, F)$, then $D \cap \Psi(\ell)=3$-space, say $\left\langle x, y, z>\right.$, where $x=x_{1}=y_{1}, y=u=y_{2}$ and $z=v=y_{3}$ at the same time take $x_{2}^{\perp} \cap<x, y, z, y_{4}, y_{5}>=<x, y, z, y_{4}>$ and $x_{3}^{\perp} \cap<x, y, z, y_{4}, y_{5}>=<x, y, z, y_{5}>$. Then we choose a point $t$ in the line $\ell$ such that $\Psi(t)=<x, y, z>$ and since $\Psi(p) \cap \Psi(t)=<x>, p$ is collinear to $t$ and $\ell \cap p^{\perp}=t$.

Theorem 3.2. Any Shult set in the geometry $D_{5,3}$ forms a geometric hyperplane.

Proof: Let $\ell$ be a line and $p$ be a point in Shult set such that $\Psi(p)=<x_{1}, x_{2}, x_{3}>$ and $\Psi(\ell)=<y_{1}, y_{2}, y_{3}, y_{4}, y_{5}>$. The proof is to find a point incident to $\ell$ and at a distant equal 4 from the point $p$, now there are three cases for $\Psi(p) \cap \Psi(\ell)$ :

1- $\Psi(p) \cap \Psi(\ell)=0$-space, then $\Psi(p)$ is contained in maximal totally isotropic 5-space, $\left\langle x_{1}, x_{2}, x_{3}, u, v>\right.$. Then there are a sequence of points $r, s$ and $t$ such that $\Psi(r)=<y_{3}, y_{4}, y_{5}>, \Psi(s)=<y_{2}, y_{3}, u>$ and $\Psi(t)=<x_{1}, u, v>$. If we choose a point $q$ incident to the line $\ell$ such that $\Psi(q)=<y_{1}, y_{2}, y_{3}>$, then

$$
\begin{aligned}
& \Psi(q) \cap \Psi(r)=<y_{3}>, \\
& \Psi(s) \cap \Psi(r)=<y_{3}>, \\
& \Psi(s) \cap \Psi(t)=<u>, \\
& \Psi(t) \cap \Psi(p)=<x_{1}>
\end{aligned}
$$

Since $<y_{1}, y_{2}, y_{3}, y_{4}, y_{5}>,<u, y_{2}, y_{3}, y_{4}, y_{5}>,<u, y_{2}, y_{3}, v, x_{1}>$ and $<x_{1}, x_{2}, x_{3}, u, v>$ are totally isotropic 5-space and $\Psi(\ell) \cap u^{\perp}=<y_{2}, y_{3}, y_{4}, y_{5}>, \Psi(\ell) \cap v^{\perp}=<y_{2}, y_{3}, y_{1}, y_{5}>$, then $q$ is collinear to $r, r$ is collinear to $s, s$ is collinear to $t$ and $t$ is collinear to $p$. This means that $\mathrm{d}(p, q)=4$.

2- $\Psi(p) \cap \Psi(\ell)=1$-space $=\langle x\rangle$, where $x=x_{1}=y_{1}$. then $\Psi(p)$ is found in a maximal totally isotropic 5-space $D=<x, x_{2}, x_{3}, u, v>$ and the points of the geodesic from $p$ to $q$ can be chosen to be $r, s$ and $t$ such that $\Psi(r)=<u, y_{4}, y_{5}>$, $\Psi(s)=<y_{5}, y_{3}, v>$ and $\Psi(t)=<x_{3}, u, v>$, where:

$$
\begin{gathered}
\Psi(q) \cap \Psi(r)=<y_{4}>, \\
\Psi(s) \cap \Psi(r)=<y_{5}>, \\
\Psi(s) \cap \Psi(t)=<v>, \\
\Psi(t) \cap \Psi(p)=<x_{3}>,
\end{gathered}
$$

If $\Psi(\ell) \cap u^{\perp}=<y_{2}, y_{3}, y_{4}, y_{5}>, \Psi(\ell) \cap v^{\perp}=<y_{4}, y_{3}, y_{1}, y_{5}>$, then $r$ is collinear to $q$ with a line is corresponding to $<y_{2}, y_{3}, y_{4}, y_{5}, u>, r$ is collinear to $s$ with a line $\left\langle u, v, y_{3}, y_{4}, y_{5}>, s\right.$ is collinear to $t$ with a line $\left\langle x_{3}, y_{3}, y_{5}, v, u>\right.$ and $t$ is collinear to $p$ with a line corresponding to $\left\langle x_{3}, x_{2}, x_{1}, v, u>\right.$. Then $\mathrm{d}(q, p)=4$, i.e., $\ell$ intersects $H$ by $q$.

3- $\Psi(p) \cap \Psi(\ell)=2$-space $=\langle x, y\rangle$, where $x=x_{1}=y_{1}$ and $y=x_{2}=y_{2}$. $\Psi(p)$ is found in a maximal totally isotropic 5-space $D=<x, y, x_{3}, u, v>$ and the geodesic between the points $p$ and $q\left(\Psi(q)=<y_{3}, y_{4}, y_{5}>, \Psi(p)=<x, y, x_{3}>\right)$ consists of the points $r, s$ and $t$ such that $\Psi(r)=<u, v, y_{5}>, \Psi(s)=<y_{4}, y_{3}, u>$ and $\Psi(t)=<x_{3}, y_{4}, v>$. Then

$$
\begin{aligned}
& \Psi(q) \cap \Psi(r)=<y_{5}>, \\
& \Psi(s) \cap \Psi(r)=<u>, \\
& \Psi(s) \cap \Psi(t)=<y_{4}>, \\
& \Psi(t) \cap \Psi(p)=<x_{3}>,
\end{aligned}
$$

If $\Psi(\ell) \cap u^{\perp}=<x, y_{3}, y_{4}, y_{5}>, \Psi(\ell) \cap v^{\perp}=<y_{4}, x_{3}, y_{3}, y_{5}>$, then $r$ is collinear to $q$ with a line is corresponding to $<v, y_{3}, y_{4}, y_{5}, u>, r$ is collinear to $s$ with a line $<u, v, y_{3}, y_{4}, y_{5}>, s$ is collinear to $t$ with a line $<x_{3}, y_{3}, y_{4}, v, u>$ and $t$ is collinear to $p$ with a line corresponding to $\left\langle x_{3}, y, x, y_{4}, v>\right.$. Then $\mathrm{d}(q, p)=4$, and the point $q$ is in Shult set.

\section{Constant-weight codes using hyperplanes}

The following definitions can be found in [Hoffman, 1992]. 
A code $C$ of length $n$ and size $M$ over a field $F$ is just a subset of $F^{n}$ of cardinality $M$, then we say that $C$ is ( $n, M$ )-code. Thus each code consists of "codewords" (vectors in $F^{n}$ ) and the number of codewords is the size of the code.

The Hamming weight of $u=\left(x_{1}, x_{2}, x_{n}\right)$ is the number of non-zero coordinates $x_{i}, i=1,2, n$, it is denoted by $w_{h}(u)$. Let $C$ be a code of length $n$ and $u, v$ be two codewords. The hamming distance between $u$ and $v, d_{h}(u, v)$, is the number of coordinate in which they differ, that is $d_{h}(u, v)=w_{h}(u+v)$. If $d=$ minimum $\left\{d_{h}(u, v): u, v \in C, u \neq v\right\}$; then $d$ is called the minimum distance of $C$, in this case we say that $C$ is $(n, M, d)$-code. If $C$ is a linear vector subspace of $F_{n}$, then $C$ is called a linear code and if the dimension of $C$ is $k$; we say that $C$ is $[n, k, d]$-code. If all codewords in $C$ have the same hamming weight $w$ then $C$ is called a constant-weight code. An $(n, M, d, w)$-code is a constant-weight $(n, M, d)$-code with $w$ as the common weight of all codewords.

The following two Propositions 4.1 and 4.2 will be used in the proof of the result in Theorem 4.3. The propositions and their proofs can be found in [Cameron P.J., 1992].

Proposition 4.1 [Cameron P.J., 1992]. The number of subspaces of dimension $k$ in a vector space of dimension $n$ over $G F(q)$ is given by the formulae:

$$
\frac{\left(q^{n}-1\right)\left(q^{n}-q\right) \cdots\left(q^{n}-q^{k-1}\right)}{\left(q^{k}-1\right)\left(q^{k}-q\right) \cdots\left(q^{k}-q^{k-1}\right)}
$$

Remark. This number in Proposition 4.2 is called a Gaussian coefficient, and is denoted by:

$$
\left[\begin{array}{l}
n \\
k
\end{array}\right]_{q}
$$

proposition 4.2. [Cameron P.J., 1992]. Let $V$ be equipped with a bilinear form then the number of totally isotropic $k$-subspaces in the hyperbolic case $\Omega^{+}(2 n, q)$ is:

$$
\left[\begin{array}{l}
n \\
k
\end{array}\right] \prod_{q}^{k-1}\left(q^{n-i-1}+1\right) .
$$

We will use the hyperplanes of type $p^{\perp}$ to construct a new family of binary constant- weight codes.

Theorem 4.3. Let $\Gamma=(P, L)$ be a point-line geometry of type $D_{5,3}$ and $H$ be a hyperplane of type $p_{i}^{\perp}, p_{i} \in P$. Let $G=\left(g_{i j}\right)$ be the incidence matrix such that

$$
g_{i j}= \begin{cases}1 & \text { if } p \in H, \\ 0 & \text { if } p \text { not in } H .\end{cases}
$$

Then the rows of $\mathrm{G}$ represent a binary constant-weight $(N, M, d, w)$ non-linear code of parameters: $N=M=P$,

$$
\begin{gathered}
w=q^{3}\left(q^{3}-1\right)\left(q^{4}-1\right), \\
d=2 w-2\left(q^{2}+1\right)^{2}(q+1)^{2}\left(q^{3}+1\right)
\end{gathered}
$$

Proof: Since $p_{i}^{\perp}$ represents a hyperplane of $\Gamma\left(p_{i} \in P\right)$, the number of rows and the number of columns of the matrix $G$ are the same number of points of $\Gamma$, so the number of code word $M$ (the size of the code) is the cardinality of $P$. Then $N=M=|P|$. Now to compute the hamming weight of the code, let $p_{j} \in P$. Then $p_{j} \in H$ if and only if $p_{j}$ is collinear to $p_{i}$ ( $H$ is a hyperplane corresponds to $p_{i}^{\perp}$ ) and that is true if $\Psi\left(p_{j}\right) \cap \Psi\left(p_{i}\right)=1$-space. So the number of points in the hyperplane $H$ is equal the number of points that are collinear to $p_{i}$ that are the number of totally isotropic 1 -space in the 3-space $\Psi\left(p_{i}\right)$ which is ( by Propositins 4.2 ) equal to

$$
\left[\begin{array}{l}
n \\
k
\end{array}\right] \prod_{q}^{k-1}\left(q^{n-i-1}+1\right) .
$$

where $n=3$ and $k=1$. Then

$$
w=\left[\begin{array}{l}
3 \\
1
\end{array}\right]_{q}\left(q^{2}+1\right),
$$




$$
\begin{gathered}
w=\frac{\left(q^{3}-1\right)\left(q^{3}-q\right)\left(q^{3}-q^{2}\right)\left(q^{2}+1\right)}{q-1} \\
w=q^{3}\left(q^{3}-1\right)\left(q^{4}-1\right) .
\end{gathered}
$$

Two rows of $G$ that correspond to the hyperplanes $p_{i}^{\perp}$ and $p_{j}^{\perp}$ have 1 in the $t^{\text {th }}$ column if the points $p_{t}$ is incident with both hyperplanes. Since any two hyperplanes intersect in a projective plane or disjoint, it follows that the corresponding two rows differ in at least $2 w-2\left|p_{i}^{\perp} \cap p_{j}^{\perp}\right|$ positions. The least of these numbers is when the two hyperplanes intersect in a projective plane, it follows that the distance is:

$$
d=2 w-2 \max \left|p_{i}^{\perp} \cap p_{j}^{\perp}\right| .
$$

Then

$$
\begin{aligned}
& d=2 w-2\left[\begin{array}{l}
4 \\
3
\end{array}\right] \prod_{q} \prod_{i=0}^{2}\left(q^{3-i}+1\right), \\
& d=2 w-2\left(q^{2}+1\right)^{2}(q+1)^{2}\left(q^{3}+1\right) .
\end{aligned}
$$

\section{References}

Abdelsalam, Z. (2007). Characterization of Dual Half-Spin Geometry. Journal of modern mathematics and statistics, 1 , $1-4$.

Bruyn, B. D. \& Pralle, H. (2007). The exceptional hyperplanes of DH(5, 4). European J. Cobinatorics, 28, 1412-1418.

Bruyn, B. D. (2007). Hyperplanes of $D W(2 n-1, q), q \neq 2$, without ovoidal quads. Discrete Math., 307, 2680-2694.

Bruyn, B. D. (2007). The hyperplanes of $D W(5,2 h)$ which arises from embedding. Discrete Math., 309, 304-321.

Buekenhout, F. \& Shult, E. E. (1974). On the foundations of polar geometry . Geometriae Dedicata, 3, 155-170.

Cameron P.J. (1992). Projective and Polar Spaces volume 13 of QMW Maths Notes. Queen Mary and Westfield College, University of London.

Cooperstein, B. N. (1977). A characterization of some Lie incidence structures . Geometriae Dedicata, 6, 205-258.

Hoffman, D. G. (1992). Coding theory II. Series, QA268.C69 .

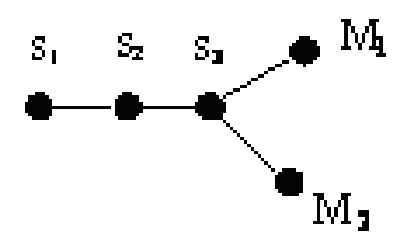

Polar space A

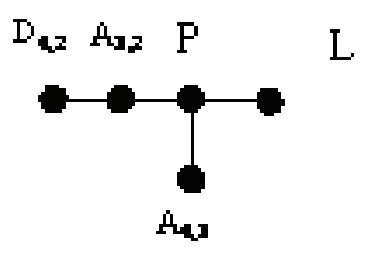

Dy,j(F)

Figure 1. 